Cuadernos de Lingüística Hispánica $n^{\circ} .23$

ISSN 0121-053X • ISSN en línea 2346-1829

Enero-Junio 2014, pp. 171-190

\title{
Formación bilingüe en licenciatura de Lenguas Modernas y comprensión lectora argumentativa*
}

\author{
ANGELMIRO GALINDO** \\ angelmirogalindo@hotmail.com \\ LINA MARÍA MORENO*** \\ linauniquindio@hotmail.com
}

* Este artículo es el resultado de una investigación científica concluida con el auspicio de la Vicerrectoría de Investigaciones de la Universidad del Q uindío, Colombia. Se inscribe en la línea de investigación Bilingüismo escolar en contextos socio-lingüísticos mayoritarios del Grupo de investigación "Estilos de Aprendizaje, Idiomas Extranjeros y Bilingüismo" [ESAPIDEX-B] de la Universidad del Q uindío, y reconocido por COLCIENCIAS.

* * Ph.D. en Lingüística, opción bilingüismo. Docente del Programa de Lenguas Modernas de la Universidad del Quindío, Colombia.

*** Magíster en Didáctica del Inglés. Docente del Programa de Lenguas Modernas de la Universidad del Quindío, Colombia. 


\title{
Resumen
}

En esta investigación se comparó la comprensión lectora argumentativa en español, lengua materna, de estudiantes en formación universitaria bilingüe de $4^{0}$ semestre de Licenciatura en Lenguas Modernas - G rupo Experimental [GE]- con la de sus homólogos en formación universitaria en LM de $4^{0}$ semestre en las Licenciaturas de Matemáticas, Español y Literatura, y Biología y Educación Ambiental -Grupo de Control [G C]-. Se halló que el GE superó al GC en la prueba de Inserción de conectores en un texto argumentativo escrito en LM y que el GE se diferenció de cada una de las tres licenciaturas. Se concluyó que la superioridad del GE sería la consecuencia de la experiencia bilingüe acumulada durante los primeros cuatro semestres de la Licenciatura.

Palabras clave: bilingüismo, formación universitaria bilingüe, conectores, comprensión lectora argumentativa.

\section{Bilingual education in the Modern Languages teaching program and argumentative reading comprehension}

\begin{abstract}
A bstract
In this research project, argumentative reading comprehension skills (in Spanish as mother tongue) of students from the Modern Languages Teaching program were compared to equivalent students of the Mathematics, Spanish and Literature, Biology en Environmental Education teaching programs. The first (Modern Languages) is the Experimental Group (EG) and the second, is the Control Group (CG). It was found that EG got better results than CG in a test about inserting connectors in a written argumentative text in Spanish; CG was remarkably different from the other three teaching programs. It could be concluded that the high performance of EG is due to the result of bilingual experience gathered through the first four semesters of the Teaching program.
\end{abstract}

Key words: Bilingualism, university bilingual education, argumentative reading comprehension. 


\section{Formation bilingue en Licence de Langues M odernes y comprehension lectrice argumentative}

\section{Résumé}

Dans cette recherche on a comparé la compréhension de lecture en séquence argumentative en espagnol langue maternelle (LM), des étudiants de quatrième semestre en formation universitaire bilingue de la Licence en Langues Modernes - G roupe Expérimental [GE]- avec celle de leurs pairs de quatrième semestre de formation universitaire des Licences de Mathématiques, Espagnol et Littérature, Biologie de l'Environnement - G roupe de Contrôle [G C]. On a déterminé que le GE a été supérieur au GC dans l'épreuve concernantl'insertion de connecteurs dans un texte écrit argumentatif en LM et que le GE s'est détaché de chacune des autres Licences. On a conclu que la supériorité du GE serait la conséquence de l'expérience bilingue accumulée pendant les trois premiers semestres de la Licence.

Mots clés: bilinguisme, formation universitaire bilingue, compréhension de lecture argumentative.

\section{$\mathbf{F}$ ormação bilíngueem licenciatura de Línguas Modernase compreensão leitora argumentativa}

\section{Resumo}

Na pesquisa se fez uma comparação leitora argumentativa em espanhol, Língua materna, dos alunos de graduação bilíngue de quarto semestre de licenciatura em Línguas Modernas - grupo experimental (GE)- com seus homólogos dos cursos de licenciatura em Matemáticas, Espanhol e Literatura, Biologia e Educação Ambiental -G rupo de Controle (GC)- . Nos resultados o GE superou ao GC na proba de inserção de conectores num texto argumentativo escrito em LM e que o GE se diferenciou de cada uma das três licenciaturas. Concluiu-se que a superioridade do $\mathrm{GE}$ seria a consequência da experiência bilíngue acumulada durante quatro semestres da Licenciatura.

Palavras chave: bilinguismo, formação universitária bilíngue, compreensão leitora argumentativa. 


\section{Introducción}

La construcción de una identidad a partir de dos lenguas-culturas, es decir, el bilingüismo, es uno de los retos a los que se enfrenta el ser humano en las nuevas sociedades de la información y de la comunicación (Abdelilah-Bauer, 2007; Baker, 2011;Chimbutane, 2011; Mueller G athercole, 2013). Estudios sobre la relación entre bilingüismo y cognición, principalmente, a partir de Peal y Lambert (1962) concluyen que el bilingüe, escolarizado en segunda lengua [L2] ${ }^{1}$, y al ser comparado con su homólogo escolarizado en su lengua materna (LM) ${ }^{2}$, tendría ventajas a nivel cognitivo, social, cultural y educativo (Grosjean, 2010; Baker, 2011; Van der Walt, 2013). D esde una perspectiva psico-educativa, el presente artículo concierne la relación entre bilingüismo y cognición, esencialmente, la comprensión lectora argumentativa en LM. En síntesis, aquí se examina dicha habilidad psicolingüística de sujetos en formación bilingüe universitaria de licenciatura en Lenguas Modernas, en comparación con sus homólogos en formación universitaria de licenciatura orientada en LM. Tal propósito investigativo exigió un estado del arte sobre la comprensión lectora en LM de sujetos en educación bilingüe y en formación universitaria orientada en LM.

\section{C omprensión lectora en lengua materna de sujetos en educación bilingüe}

En este apartado se presenta un breve estado de la cuestión sobre la comprensión lectora en LM de beneficiarios de la educación bilingüe,

\footnotetext{
La segunda lengua hace referencia a la lengua hablada en la comunidad en la cual se encuentra el aprendiz de la lengua. Por ejemplo, el inglés es una segunda lengua para un inmigrante mexicano en Estados Unidos (Muñoz, 2002).

2 El concepto de lengua materna [LM] se refiere, normalmente, al código lingüístico que el sujeto utiliza como primer sistema de comunicación y, por lo demás, se adquiere en el hogar. Es, igualmente, el código lingüístico utilizado como LM por la mayoría de los miembros de una comunidad lingüística (Hamers y Blanc, 2000).
} 
principalmente, por inmersión en varios contextos socioeducativos del mundo. El primero corresponde al de la Comunidad Autónoma Vasca de España, en donde conviven como lenguas oficiales el castellano y una lengua minoritaria, el euskera o vasco. Según Sierra (1993), al inicio del ciclo de primaria, los alumnos castellanoparlantes de los dos modelos de educación bilingüe existentes en el país Vasco ${ }^{3}$, al ser comparados con sus homólogos escolarizados en LM, manifiestan un cierto retraso en comprensión de lectura. Sin embargo, afirma Sierra que al finalizar el ciclo de primaria, los beneficiarios del sistema educativo bilingüe, en comparación con sus homólogos escolarizados en LM, obtienen el mismo nivel de rendimiento en las pruebas de comprensión de lectura en LM, entre otras: 1) Identificación de la idea principal y 2) Reconocimiento de los argumentos que la sustentan. Sierra concluye que aún la enseñanza en L2 (euskera) más intensiva no produce en los beneficiarios de la educación bilingüe ningún detrimento de su LM y, en particular, la comprensión de lectura.

El segundo contexto de educación bilingüe, aquí referenciado, corresponde al de la Comunidad Autónoma Española de Cataluña. En este contexto socioeducativo, Trueta, Barrachina y Pascual (2012) analizan las evaluaciones PISA (Programme for International Student Assessment) de 2009, con el fin de determinar el rendimiento en comprensión lectora de los alumnos catalanes, al finalizar la primaria, 0 sea, el grado $6^{0}$. Los resultados se interpretan utilizando criterios de comparación interna entre alumnos del mismo contexto, y externa con alumnos en contextos educativos convencionales. D e la comparación interna, los investigadores concluyen que la comprensión lectora de los alumnos en programas de inmersión es superior a la de alumnos que aprenden en la LM. De la comparación externa, los autores concluyen que los alumnos catalanes, en su mayoría en programas de inmersión, se sitúan en una posición intermedia (posición 15/35), y muy por encima del Estado español (posición 28/35), con un porcentaje importante de población monolingüe y asistiendo a programas monolingües en castellano.

El breve estado de la cuestión, presentado en esta sección, sobre la comprensión lectora en LM en contextos educativos bilingües concluye con datos provenientes de los contextos educativos por inmersión en L2 en Canadá y en

\footnotetext{
Los dos modelos de educación bilingüe del país Vasco están dirigidos a niños castellanoparlantes y, por lo demás, se clasifican en B y D. En el Modelo B, se imparte la enseñanza en euskera (L2) y castellano (LM), en una especie de inmersión parcial temprana. En el Modelo D, la enseñanza es en euskera (L2) y el castellano (LM) constituye una asignatura, en una especie de inmersión total temprana (Sierra, 1993).
} 
lengua extranjera [LE $]^{4}$ en Colombia. En Canadá, Genesee (2004), G enesee y G eva (2006) y G enesee y Jared (2008) encontraron que en los grados primero y segundo de primaria en inmersión precoz total y parcial en francés los sujetos en inmersión son menos competentes que sus homólogos escolarizados en LM en la prueba de lectura English-Language Testing of Literacy Skills, cuyos componentes son, entre otros: comprensión de lectura, ortografía y comprensión de vocabulario. Sin embargo, afirman los autores anteriores, que a partir del grado $3^{\circ} 0$ 4ํㅡㄹ de primaria, cuando la LM es introducida al programa bilingüe, los niveles de lectura en LM de los bilingües son similares a los de sus homólogos escolarizados en LM. Paralelamente, tal similitud se observa en el último grado de la escolaridad primaria (6og grado) en inmersión en L2 (Turnbull, Lapkin \& Hart, 2001). Seguidamente, en la educación bilingüe por inmersión en francés en los primeros grados de la secundaria en Canadá, Allen (2004) reporta que los bilingües anglófonos de 15 años de edad son más competentes que sus homólogos escolarizados en su LM (inglés) en comprensión lectora en las pruebas PISSA de 2003, específicamente, en actividades de interpretación, análisis de información y reflexión sobre la forma y contenido del texto. Entonces, los beneficiarios de dichos programas de inmersión, tanto en la educación primaria como secundaria, en comparación con sus homólogos escolarizados en LM, no estarían en desventaja en comprensión lectora en LM.

La comprensión lectora en LM de sujetos en inmersión en lengua extranjera en Colombia es una línea de investigación exploratoria. Los datos relacionados a continuación reflejan la comprensión lectora en LM de sujetos en inmersión precoz parcial en inglés como LE. Galindo (2009) concluyó que estudiantes hispanohablantes colombianos de último grado de primaria (5ํao gra) en inmersión precoz parcial en inglés obtuvieron resultados análogos a los de sus homólogos escolarizados en español/ LM en pruebas de lectura, tales como: preguntas de selección múltiple con única respuesta y preguntas dicotómicas. Partiendo del modelo psicolingüístico de la hipótesis de los umbrales de Cummins (2005), G alindo sustenta dicha similitud en el hecho de que estos bilingües habrían alcanzado un primer umbral de competencia lingüística. Entonces, sin pretender exhaustividad, los datos relativos a la comprensión lectora en LM de sujetos en inmersión en LE irían en la misma tendencia de los datos relacionados

Se habla de lengua extranjera (LE) cuando una lengua no es LM ni lengua propia del país en que se estudia 0 aprende, es decir, es lengua de otro país. Igualmente, la lengua extranjera se refiere a aquella que se aprende en un contexto donde no cumple una función social e institucional(Muñoz, 2002).

\section{6}


anteriormente, provenientes de la educación bilingüe por inmersión en segunda lengua. En tal sentido, el modelo de educación bilingüe por inmersión en segunda lengua y en lengua extranjera beneficiaría el desarrollo lingüístico del sujeto y, en particular, la comprensión escrita en LM.

\subsection{Comprensión lectora de sujetos en formación universitaria orientada en LM}

El estado del arte, referenciado seguidamente, sobre la comprensión lectora en LM de sujetos en formación universitaria orientada en LM proviene de dos fuentes: la primera, de investigaciones universitarias colombianas, y la segunda, de evaluaciones sobre comprensión lectora de estudiantes universitarios próximos a la graduación, realizadas por el Estado de este país.

La investigación sobre la comprensión lectora en LM en el contexto universitario de Colombia involucra la realizada en la Universidad Colegio Mayor de Cundinamarca y la Universidad Cooperativa de Colombia. En la primera universidad, caracterizada por la implementación en sus estudiantes de la lectura crítica, Andrade Calderón (2007) realizó una investigación descriptiva-cualitativa con una muestra poblacional de 1.314 estudiantes. Sus hallazgos fueron: 1) La mitad de la población encuestada está en un rango de lector con "comprensión mediana" y 2) Las dificultades en comprensión lectora de estos estudiantes se sitúan en dos niveles: textual y pragmático. Las del primer nivel tienen que ver con la identificación del lenguaje, tipo de discurso y reconocimiento de la estructura del texto. Las del segundo nivel conciernen a la manifestación creativa y la identificación de la intencionalidad del texto. En la segunda universidad, es decir, la Universidad Cooperativa de Colombia, seccional de Barrancabermeja, Calderón Ibáñez y Q uijano Peñuela (2010) realizaron un estudio descriptivo sobre la lectura con estudiantes de último año en las áreas de psicología y derecho, por medio de pruebas de tipo doze Sus resultados fueron: 1) No hay manejo gramatical del lenguaje entendido como competencia lingüística por la aplicación acertada de la sintaxis de la lengua, la fonética y la semántica en las micro y macroestructuras, y 2) No se evidencia en los estudiantes el paso por los distintos niveles de adquisición, retención, integración, recuperación y transferencia de información. Según estos investigadores, los encuestados se hallan en un nivel de comprensión literal y su léxico es muy reducido.

Los datos del Estado Colombiano sobre la valoración de la comprensión lectora de estudiantes en formación universitaria orientada en LM, provienen 
del Examen de Estado de Calidad de la Educación Superior [SABER PRO] ${ }^{5}$, el cual evalúa las competencias genéricas y específicas. Hace parte de las pruebas de competencias genéricas la de Lectura oútica A continuación se hace énfasis en los resultados de dicha prueba, obtenidos en 2011 y 2012 en instituciones universitarias.

En la aplicación de las pruebas SABER PRO 2011, participaron $14^{6}$ programas de universidades colombianas. Según el Instituto Colombiano para la Evaluación de la Educación [ICFES] ${ }^{7}$, la distribución de la proporción correspondiente a los desempeños en lectura crítica es la siguiente: Medicina obtuvo el porcentaje más alto (47\%) de mejores desempeños, y Educación obtuvo el porcentaje más alto (28\%) de bajos desempeños. Los resultados en la prueba de Lectura Crítica del $2012^{8}$ indican que los egresados de los programas de las facultades de Educación obtienen los resultados más bajos y los egresados de las facultades de Medicina, los más altos. Seguidamente, en los estudiantes de los programas de Educación que presentaron la prueba de Lectura Crítica en este mismo año, la distribución del número de estudiantes ubicados en cada uno de los quintiles ${ }^{9}$ de la prueba es la siguiente:

\begin{tabular}{|c|c|c|c|c|c|}
\hline Total estudiantes & Q1 & Q2 & Q3 & Q4 & Q 5 \\
\hline 21.308 & 5.038 & 4.086 & 3.886 & 4.962 & 3.336 \\
\hline
\end{tabular}

La tabla anterior revela que en el Q1 y Q 2 están ubicados los estudiantes clasificados como lectores deficientes. En otras palabras, 9.124 estudiantes (42.8\%) no habrían desarrollado las competencias y habilidades necesarias para leer un texto de manera crítica y reflexiva. Igualmente, de la población encuestada, 3.886 estudiantes, es decir, el 18.2\% alcanza un nivel intermedio (Q 3) en lectura crítica. Adicionalmente, la sumatoria del número de estudiantes del Q4 y Q 5

Las Pruebas Saber Pro se aplican a los estudiantes de último año en los distintos programas de las instituciones universitarias y no universitarias.

6 Los 14 programas universitarios son: 1) Arquitectura y Urbanismo, 2) Bellas Artes y Diseño, 3) Comunicación, periodismo y Publicación, 4) Ciencias Agropecuarias, 5) Ciencias Económicas y Administrativas, 6) Ciencias Militares y Navales, 7) Ciencias Naturales y Exactas, 8) Ciencias Sociales, 9) Derecho, 10) Educación, 11) Humanidades, 12) Ingeniería, 13) Medicina y 14) Salud.

www.icfes.edu.co

8 ICFES (Junio 14 de 2013).Resultados agregados de saber pro en los módulos de competencias genéricas, año 2012. Programas Universitarios. Recuperado de http:/ / www.icfes.gov.co/ resultados/

9 El quintil 1 (Q1) corresponde al 20\% de los evaluados con los puntajes más bajos y el quintil 5 (Q5) al 20\% de los evaluados con los puntajes más altos. 
equivale a 8.298 , lo que indica que el $38.9 \%$ estarían considerados como buenos lectores. Finalmente, 3.336 estudiantes, esto es, el 15.7\% desarrollaron las competencias y habilidades de comprensión lectora necesarias para ser considerados como muy buenos lectores. Entonces, estos datos indican que a noviembre de 2012, el 42.8\% de los estudiantes a graduarse en las facultades de Educación de Colombia no sabrían leer críticamente.

D el estado del arte, presentado anteriormente, sobre la comprensión lectora en LM de sujetos en educación bilingüe por inmersión y en formación universitaria orientada en LM emergerían dos conclusiones. Primero, la educación bilingüe no resultaría perjudicial para el desarrollo de la comprensión lectora en LM de sus beneficiarios, ya que estos, principalmente, al finalizar la primaria y en los primeros grados de la secundaria, son tan competentes e, incluso, superiores a sus homólogos escolarizados convencionalmente. Segundo, del sistema universitario colombiano orientado en LM se graduarían en los años 2011 y 2012 profesionales que deben mejorar su desempeño en lectura en LM.

Por otra parte, si al finalizar la escolaridad primaria bilingüe y en los primeros grados de la secundaria bilingüe, sus beneficiarios son tan competentes e, incluso, superiores en comprensión lectora en LM a sus homólogos escolarizados en LM (Allen, 2004; G enesee, 2004; G enesee y G eva, 2006; Genesee y Jared, 2008; Galindo, 2009), resultaría lógico hipotetizar que suceda lo mismo en niveles superiores de formación escolar bilingüe; por ejemplo, el universitario de Licenciatura en Lenguas Modernas. En tal dirección, se tendría la siguiente hipótesis de investigación de diferencia entre grupos: los estudiantes en formación bilingüe universitaria de Licenciatura en Lenguas Modernas, específicamente de $4^{0}$ semestre, [G rupo experimental, GE] obtienen mejores desempeños en comprensión lectora argumentativa en LM que sus homólogos en formación universitaria orientada en LM; por ejemplo, en las licenciaturas de Matemáticas, Español y Literatura, y Biología y Educación Ambiental [G rupo de control, GC] ${ }^{10}$. De lo anterior se desprende la pregunta de investigación siguiente:¿qué diferencias 0 similitudes existen entre estudiantes universitarios en formación bilingüe de $4^{0}$ semestre de Licenciatura en Lenguas Modernas [GE] y sus homólogos en

El grupo de control [GC] es el grupo que no está expuesto a la influencia de la variable independiente (la escolarización por medio de la inmersión en segunda lengua). Se decidió conformar el GC con sujetos de estas tres licenciaturas porque están en formación de pregrado en la misma área general del conocimiento del grupo experimental [GE], es decir, la educación. A simismo, al igual que los sujetos del GE, están siendo formados para el ejercicio de la docencia en educación básica y media, el cual les exige habilidades de comprensión lectora en general y, en particular, argumentativa. 
formación universitaria orientada en LM en las licenciaturas en Matemáticas, Español y Literatura, y Biología y Educación Ambiental [G C] en cuanto a la comprensión lectora argumentativa en LM?

\section{Metodología}

\subsection{M uestra poblacional}

Esta investigación ${ }^{11}$ se realizó con dos grupos de estudiantes hispanohablantes colombianos. El primero, en formación bilingüe universitaria, Grupo Experimental (GE) y, el segundo, en formación universitaria en LM, G rupo de Control (GC). El GE lo conformaron 26 sujetos que culminan el 4/ 10 semestre $^{12}$ de Licenciatura en Lenguas Modernas. Las condiciones de promoción del bilingüismo de este grupo fueron las siguientes: primero, aprendizaje del inglés como lengua extranjera, desarrollando habilidades en gramática, comprensión, producción escrita y oral, durante los primeros cuatro semestres de la licenciatura, a razón de 15 horas semanales, para un total acumulado de 960 horas. Del mismo modo, estos sujetos inician el primer semestre de la licenciatura a los 17.5 años de edad aproximadamente. Es, entonces, un caso de bilingüismo individual consecutivo en entornos universitarios.

El GC estuvo compuesto por 35 sujetos que culminan el 4/ 10 semestre de licenciatura orientada en español/ LM en las áreas de Matemáticas, Español y Literatura, y Biología y Educación Ambiental. Este grupo recibe las asignaturas que conforman el plan de estudios de las respectivas licenciaturas en su LM español-. Adicionalmente, los sujetos de cada una de las licenciaturas que conforman el GC cursaron y aprobaron la asignatura en inglés como lengua extranjera durante los tres primeros semestres del plan de estudios, a razón de 2 horas semanales, para un total acumulado de 96 horas. El objetivo general de esta asignatura durante el ciclo de los tres semestres consistió en desarrollar habilidades de comprensión lectora, en general, de textos de información propia de cada licenciatura; en otras palabras, no se hizo énfasis en el desarrollo de habilidades de comprensión lectora de textos argumentativos. Debido a este entrenamiento en lengua extranjera, resultaría lógico hipotetizar que tales sujetos

${ }_{11}$ En este estudio no se manipuló intencionalmente la variable independiente, sino que se observó en su estado natural para, posteriormente, analizarla en relación con la variable dependiente. Igualmente, aquí se comparó el GE con el GC en relación con la variable dependiente. Entonces, el diseño de investigación es no experimental, causal y comparativo.

12 El semestre académico tiene un total de 16 horas. 
poseerían un estado de bilingüismo asimétrico, principalmente, en el nivel escrito $^{13}$.

La formación lingüística universitaria en LM del GE y GC se describe de la siguiente manera. Como parte de las Actividades Obligatorias de Ley (AOL), el GE y el GC recibieron un entrenamiento en español/ LM. ElG E cursó y aprobó la asignatura de Español I, II, III, IV, con una intensidad horaria semanal de 4 horas. En los tres primeros cursos se desarrollan habilidades de expresión oral, de lectura y escritura general. El curso de español IV tiene como finalidad el desarrollo de habilidades de lectura y escritura de textos de naturaleza argumentativa. D e forma paralela a las actividades académicas profesionales y básicas, los sujetos de cada licenciatura del GC cursaron y aprobaron la asignatura de Español I y II durante el primero y segundo semestres de cada licenciatura, a razón de 4 horas semanales. El objetivo de estos cursos consistió en desarrollar habilidades en expresión oral, de lectura y escritura general, es decir, no se hizo énfasis en el desarrollo de habilidades de comprensión de lectura de textos argumentativos.

\subsection{Prueba de comprensión lectora argumentativa}

La comprensión lectora argumentativa fue medida mediante la Prudba de inserción deconetores en un texto argumentativo escitoen españd/ LM, la cual fue una adaptación del modelo experimental propuesto por Akiguet y Piolat (1996). El estudiante recibe un texto argumentativo escrito en español/ LM, en el cual los conectores (marcadores de relaciones textuales) han sido suprimidos y reemplazados por una línea de longitud constante. Su tarea consiste en llenar cada espacio en blanco con el conector que estime conveniente (el estudiante no tiene a su disposición ni diccionarios, ni lista de conectores).

\subsection{Procedimiento de recolección de datos y de corrección de la prueba de comprensión lectora argumentativa}

El control o equivalencia de la muestra poblacional se llevó a cabo como sigue: primero, se tuvo en cuenta que el GE y GC fueran equivalentes en todo, excepto en la presencia de la variable independiente. La equivalencia en los grupos se logró mediante la manipulación de variables de control14. Los grupos

${ }_{13}$ El sujeto que posee un estado de bilingüismo asimétrico en lo escrito podría leer y escribir en la segunda lengua, pero no podría usarla para fines de comprensión y de expresión del oral (Grosjean, 2010; Baker, 2011).

14 Aquí se construyó un instrumento de control de la muestra poblacional. Fue administrado colectivamente y respondido por los sujetos individualmente. Su administración tuvo lugar antes de la administración del instrumento de medición de la variable de control. 
fueron apareados en relación con la edad: el promedio del GE equivale a19.5 años y el del G C a 19.8 años. Por último, se tuvo en cuenta que ningún sujeto del $\mathrm{GE} \mathrm{y} \mathrm{GC}$ fuera considerado como bilingüe funciona ${ }^{15}$ antes de iniciar los estudios de la respectiva licenciatura y que, por lo demás, ninguno de ellos hubiera recibido entrenamiento en lectura por fuera del contexto universitario.

Se administró la prueba de comprensión lectora argumentativa en español/ $\mathrm{LM}^{16}$ en el GE y GC. Con la debida tramitación de los requerimientos administrativos, la recolección de datos de la variable dependiente en el GE se llevó a cabo antes de que este hubiera cursado la asignatura de Español IV. Se procedió así para evitar que los datos fueran contaminados por tal entrenamiento y que el GC estuviera en desventaja con el GE, ya que el G C no tuvo un curso de español en el desarrollo explícito de este tipo de comprensión lectora. Paralelamente, la aplicación de esta prueba fue colectiva, mediante la técnica de autoaplicación. Su resolución fue individualmente. Por último, la técnica de corrección de la prueba fue la siguiente. La respuesta del estudiante es correcta, si el conector propuesto corresponde al del texto original (D e Landsheere, 1973) o si es un sinónimo de este (Weir, 1990). Entonces, a cada respuesta correcta se le asigna un (1) punto; en todo caso contrario, se le asigna cero (0) puntos. El nivel de acierto del estudiante a esta prueba se obtuvo sumando el número de respuestas correctas.

\section{Presentación y discusión de resultados}

Los resultados ${ }^{17}$ se presentan y se discuten en dos direcciones. La primera trata de las diferencias o similitudes entre el GE (estudiantes universitarios en formación bilingüe de $4^{0}$ semestre de Licenciatura en Lenguas Modernas) y el GC (estudiantes de 4ํㅗ semestre de licenciatura orientada en español/ LM en las áreas de Matemáticas, Español y Literatura, y Biología y Educación Ambiental) en cuanto la puntuación global de la prueba de inserción de conectores en un

${ }_{15}$ Partiendo del modelo de escalas de autoevaluación del bilingüismo funcional propuesto por Baker (2011), se construyó una escala ordinal para medir el uso actual de dos lenguas en el sujeto en cada una de las cuatro habilidades lingüísticas. A cada respondiente se le pidió que manifestara su grado de uso del español (LM) y de otra lengua (inglés 0 francés) en una escala de 1 a 5 (1 = Siempre en español, $2=$ Con más frecuencia en español que en otra lengua, 3 = Con la misma facilidad en español y en otra lengua, $4=$ Con más frecuencia en otra lengua que en español y $5=$ Siempre en otra lengua). Dicho instrumento reunió los criterios de confiabilidad y validez.

16 El nivel de dificultad de la prueba fue verificado en un grupo piloto, cuyos resultados indicaron que el $87 \%$ de los sujetos la resolvieron acertadamente.

17 En la prueba de hipótesis del tratamiento estadístico de los resultados se utilizó un nivel de significancia (valor-p) equivalente a 0.05 . 
texto argumentativo escrito. La segunda dirección contiene análisis estadísticos secundarios atinentes a las diferencias o semejanzas en dicha prueba entre el GE y cada especialidad de licenciatura del GC.

\subsection{Resultados del GE y G C en la puntuación global dela prueba de inserción deconectores en un texto argumentativo escrito en LM}

Tabla 1. Comparación de promedios del GE y GC en la puntuación global de la prueba de inserción de conectores en un texto argumentativo escrito

\begin{tabular}{|c|c|c|c|c|c|}
\hline Grupo & $\mathrm{N}$ & $\begin{array}{c}\text { Media } \\
\text { (valor máximo: } \\
11 \text { puntos) }\end{array}$ & $\mathrm{DE}$ & $\begin{array}{c}\text { Prueba de } \\
\text { valor } \\
\text { estadístico }\end{array}$ & $\begin{array}{c}\text { Kruskal-Wallis } \\
\text { valor-p }\end{array}$ \\
\hline GEG & 2635 & 7.055 .28 & 2.012 .06 & 9,00698 & 0,00268854 \\
\hline
\end{tabular}

La tabla anterior revela que el promedio del GE es superior al del GC. Este hallazgo confirma la hipótesis de la diferencia entre grupos de la presente investigación: los estudiantes en formación bilingüe universitaria de $4^{0}$ semestre de Licenciatura en Lenguas Modernas [GE] obtienen mejores desempeños en comprensión lectora argumentativa en LM que sus homólogos en formación universitaria en LM en las licenciaturas de Matemáticas, Español y Literatura, y Biología y E ducación Ambiental [GC]. D ado que el G E pertenecería a la tipología de bilingüismo consecutivo en contexto educativo, su superioridad sobre el GC se explicaría desde el modelo psicolingüístico de la hipótesis del umbral (Cummins, 2005). Según esta hipótesis, los beneficios cognitivos del bilingüismo solo se alcanzan cuando el sujeto supera un segundo umbral de competencia lingüística ${ }^{18}$. A simismo, en el contexto de este modelo psicolingüístico, y dada la superioridad del GE sobre el GC en la comprensión escrita argumentativa, se podría sugerir que el $G \mathrm{E}$ habría desarrollado el bilingüismo aditivo. Sin embargo, no se pretende universalizar la anterior constatación. Se requiere, obviamente, de otras investigaciones en tal dirección.

En la tabla anterior, se observa, igualmente, que la desviación estándar (DE) de ambos grupos es alta. Este análisis estadístico indicaría que existe mucha dispersión de datos, es decir, variabilidad. En términos de desempeño en la comprensión lectora argumentativa, significaría que, en ambos grupos, existen

${ }_{18}-\bar{L}$ La competencia lingüística hace referencia al dominio del instrumento lingüístico y no a aspectos específicamente lingüísticos, como: morfosintaxis, pronunciación, etc. (Cummins, 2005). 
algunos lectores con puntuaciones muy altas, y otros con puntuaciones muy bajas. Sin embargo, la heterogeneidad es mayor en el GC. En otros términos, tanto en el GE como en el GC habría estudiantes que revelan dificultades en la comprensión de la función argumentativa de los conectores utilizados en un texto argumentativo escrito en LM. Dificultad que pudiera estar asociada a diversos factores, entre otros: 1) El manejo gramatical del lenguaje, entendido como la competencia lingüística por la aplicación acertada en las micro y macroestructuras del texto (Calderón Ibáñez y Q uijano Peñuela, 2010) y 2) La aplicación acertada de estrategias de comprensión lectora de orden cognitivo (reconocimiento y comprensión de palabras, frases, etc.) y metacognitivo (activación de conocimientos previos, determinación de la finalidad de la lectura, etc.) (Efklides, 2009). Seguidamente, si se tiene en cuenta que en la presente investigación se aborda la comprensión lectora como producto, entendido desde un enfoque cognitivo, como la resultante de la interacción crítica y creativa entre el lector y el texto y dada la alta dispersión en el GE y GC, se concluye que ambos grupos manifestarían dificultades en la comunicación en LM, sobre todo si se considera que la lectura - al lado de la escritura- es un elemento fundante de dicha comunicación (Cassany, 2006). Nótese, al mismo tiempo, que tal comunicación es una de las competencias clave para la realización y desarrollo personal del ser humano ${ }^{19}$.

Las altas desviaciones estándares del GE (M = 7.05, DE $=2.01)$ y GC $(\mathrm{M}=5.28, \mathrm{DE}=2.06)$ indicarían, entonces, que la competencia lectora argumentativa en LM es para estos sujetos una actividad cognitiva compleja, particularmente, en la comprensión de la función argumentativa de los conectores y de su respectivo uso (inserción) en el texto en lectura. En realidad, desde las aportaciones de la psicolingüística y de la teoría del procesamiento de la información, se asume que la lectura en general "es una actividad compleja que parte de la decodificación de los signos escritos y termina en la comprensión del significado de las oraciones y los textos"(Morais, 2001).

Para terminar, estudios realizados en torno de la comprensión lectora en LM de sujetos en inmersión en segunda lengua en primaria (Turnbull y otros,

${ }_{19}{ }^{-}$Las demás competencias, según el marco común de referencia de la Unión Europea del 2006, son: 1) Competencia en comunicación lingüística, 2) Competencia matemática y competencias básicas en ciencia y tecnología, 3) Competencia digital, 4) Aprender a aprender, 5) Competencias sociales y cívicas, 6) Sentido de iniciativa y espíritu de empresa y 7) Conciencia y expresión culturales. Recuperado de http:/ / eurlex.europa.eu/ LexUriServ/ LexUriServ.do?uri=CELEX:32006H0962:E S:NOT 
2001; G enesee, 2004; Genesee y G eva, 2006; Genesee y Jared, 2008) y en secundaria (Allen, 2004), así como en sujetos en inmersión en lengua extranjera en primaria ( $G$ alindo, 2009) permitirían entrever que estos modelos de educación bilingüe beneficiarían el desarrollo de dicho aspecto de uso del lenguaje. El presente estudio reveló que los bilingües en formación bilingüe en el ciclo universitario obtuvieron mejores desempeños en la comprensión lectora argumentativa que sus homólogos en formación universitaria en LM. Entonces, esta evidencia en su conjunto sugiere tres aspectos: 1) El efecto positivo de la formación escolar bilingüe comienza desde los últimos grados de la primaria por inmersión, 2) En los primeros grados de secundaria de inmersión se observa el mismo efecto y, finalmente, 3) En sujetos en formación bilingüe universitaria de pregrado en Lenguas Modernas se observaría la misma tendencia.

\subsection{Resultados del GE en relación con cada especialidad de licenciatura del GC en la inserción de conectores en un texto argumentativo escrito en LM}

Los datos se analizaron aquí con la finalidad de determinar en qué medida la comprensión lectora argumentativa en LM de los sujetos en formación bilingüe universitaria de $4^{0}$ semestre de Licenciatura en Lenguas Modernas difiere 0 se asemeja a la de sus homólogos de cada especialidad de licenciatura del GC.

Tabla 2. Comparación entre el GE y cada especialidad de licenciatura orientada en LM del GC en la puntuación global de la prueba de Inserción de conectores en un texto argumentativo escrito

\begin{tabular}{|c|c|c|c|c|c|c|}
\hline Tipo de pregrado & $\mathrm{N}$ & $\begin{array}{c}\text { Media } \\
\text { (Valor máximo: } \\
11 \text { puntos) }\end{array}$ & D E & \multicolumn{3}{|c|}{$\begin{array}{c}\text { G rupos } \\
\text { homogéneos }\end{array}$} \\
\hline Matemáticas & 11 & 5.68 & 2.23 & & $\mathrm{X}$ & \\
\hline Biología y Educación Ambiental & 12 & 5.86 & 1.74 & $\mathrm{X}$ & $\mathrm{X}$ & \\
\hline Español y Literatura & 12 & 4.42 & 1.86 & & $\mathrm{X}$ & $\mathrm{X}$ \\
\hline Lenguas Modernas (GE) & 26 & 7.05 & 2.01 & & & $\mathrm{X}$ \\
\hline
\end{tabular}

En la tabla anterior se observa que los sujetos bilingües de la licenciatura en Lenguas Modernas (GE) fueron superiores a sus homólogos de cada una de las licenciaturas del GC. Igualmente, se observa que los sujetos de las licenciaturas en Lenguas Modemas y en Matemáticas fueron superiores a sus homólogos de las 
licenciaturas en Biología y Educación Ambiental y los de Español y Literatura. Se observa, por último, que los sujetos de la licenciatura en Biología y Educación Ambiental y los de la licenciatura en Español y Literatura fueron similares entre sí.

La superioridad del GE sobre los sujetos de cada una de las licenciaturas orientadas en LM podría estar asociada a la superioridad del GE sobre el GC hallada en la comparación de la prueba de inserción de conectores en el texto argumentativo (ver la Tabla 1). D icha superioridad sería, igualmente, atribuible al logro del segundo umbral de competencia lingüística (Cummins, 2005). Paralelamente, teóricos de la argumentación, entre ellos, Perelman y OlbrechtsTyteca (1958/ 1992), Charaudeau (1992) y Thyrion (1997) sostienen que la comprensión acertada de la función de los conectores argumentativos ayudaría a una mejor elaboración del discurso argumentativo. Según estos teóricos, tal comprensión exigiría un procedimiento psicolingüístico consciente del sujeto argumentador, en este caso, lector del texto argumentativo. Desde este breve marco explicativo de la argumentación, resultaría lógico suponer que la superioridad de los sujetos en formación bilingüe sobre sus homólogos de cada una de las licenciaturas en LM sería atribuible al hecho de que los bilingües habrían alcanzado una mayor comprensión de la naturaleza de los conectores argumentativos y, que por lo demás, serían más conscientes de dicho procedimiento.

D esde una perspectiva psicolingüística del bilingüismo se ha demostrado que los sujetos en formación escolar bilingüe, en comparación con sus homólogos en formación escolar en LM tendrían un mayor control ejecutivo en actividades de uso de lenguaje, entre otras, emisión de juicios sobre la gramaticalidad del lenguaje y comprensión de la relación simbólica existente entre las palabras escritas y su significado (Bialystok, Luk, Peets, \& Yang, 2010). Dicho control ejecutivo podría ser transferido a otras actividades de uso del lenguaje, en este caso, la comprensión de la función argumentativa de los conectores. En el marco de esta hipótesis, los bilingües de la licenciatura en Lenguas Modernas, al ser comparados con los sujetos de cada licenciatura en LM, habrían movilizado un mayor control ejecutivo en la tarea realizada.

\section{Condusiones}

En el presente artículo, la comprensión lectora argumentativa en español/ LM fue evaluada por medio de la prueba de inserción de conectores en un texto argumentativo en español/ LM. Los datos se analizaron en torno de las diferencias 
o similitudes entre estudiantes universitarios en formación bilingüe de $4^{0}$ semestre de Licenciatura en Lenguas Modernas y sus homólogos en formación universitaria en LM en las licenciaturas en Matemáticas, Español y Literatura, y Biología y Educación Ambiental. Igualmente, se analizaron para determinar si existían diferencias o similitudes entre el GE y cada una de las licenciaturas del GC.

Los estadísticos sobre las diferencias entre los grupos indicaron que el GE fue superior al GC y a cada una de las licenciaturas orientada en LM. D esde una perspectiva psicolingǘstica del bilingüismo, principalmente, de la correlación positiva entre bilingüismo y cognición es posible sugerir que el GE tendría un rango de experiencias lingüísticas más amplio y variado que el $\mathrm{GC}$, debido a que está inmerso en el uso de dos lenguas y culturas diferentes. Esta condición le permitiría, consciente 0 inconscientemente, comparar y contrastar sus dos lenguas en diferentes dimensiones, entre otras, la comprensión del significado y de la organización gramatical. Estas condiciones podrían desarrollar en el GE una mayor experiencia en el lenguaje, principalmente, la comprensión escrita argumentativa, que lo facultaría para un mejor desempeño que el GC en la prueba de inserción de conectores en el texto argumentativo escrito. Igualmente, dicha superioridad sería la consecuencia de la formación bilingüe acumulada durante los primeros cuatro semestres de la Licenciatura en Lenguas Modernas. En este periodo, los bilingües alternarían entre sus dos lenguas, lo que les aseguraría una mayor flexibilidad cognitiva, con beneficio lateral positivo en la comprensión de los conectores argumentativos. Entonces, el efecto positivo de la formación bilingüe en contexto educativo, observado en los niveles de primaria y secundaria (Turnbull y otros, 2001; Genesee, 2008; G alindo, 2009; Trueta, Barrachina y Pascual, 2012) se extiende a la formación bilingüe en el contexto educativo superior, en este caso, la Licenciatura en Lenguas Modernas.

Los estadísticos sobre las similitudes entre el GE y GC revelaron altas desviaciones estándares de sus promedios, indicando que la comprensión de la función de los conectores argumentativos y, por lo demás, su uso en el texto argumentativo escrito sería para ambos grupos una actividad lógico-discursiva compleja. Con el fin de propiciar mejores desempeños en tal aspecto en ambos grupos, se recomendaría la implementación de un enfoque didáctico metacognitivo, que involucre al estudiante universitario en el uso auto-regulado del discurso argumentativo escrito. En tal sentido, se recomendarían, entre otras estrategias metodológicas, las siguientes: el análisis metadiscursivo y metalingǘstico de la manera como se componen y funcionan los diferentes tipos 
de discursos argumentativos, los ejercicios de reconstrucción textual, la composición y decodificación de textos(Padilla y otros, 2011).

Finalmente, los resultados de la presente investigación sustentan la hipótesis de la correlación positiva entre bilingüismo y cognición, principalmente, el bilingüismo consecutivo de la edad adulta en entornos universitarios de Licenciatura en Lenguas Modernas y la comprensión lectora argumentativa. Esta es una línea de investigación exploratoria, principalmente, en Colombia. Futuras investigaciones serían necesarias para replicar los datos aquí hallados, involucrando otras variables de la comprensión lectora argumentativa, entre ellas, elaboración de resúmenes, construcción del sentido global de un texto, establecimiento de relaciones entre sus enunciados y evaluación de su intencionalidad.

\section{Referencias bibliográficas}

Abdelilah-Bauer, B. (2007). El desafío de bilingüism Creer y vivir hablandb varios idiomas Madrid: Ediciones Morata.

Akiguet, S. \& Piolat, A. (1996). Insertion of connectives by 9- to 11- year-old children in an argumentative text. En: Argumetation Vol. 10. pp. 253-270.

Allen, M. (2004). Reading achievement of students in French immersion programs. En: Edurational Quartely Review Vol. 9,No4. pp. 25-30.

Andrade Calderón, M. (2007). La lectura en los universitarios. Un caso específico: Universidad Colegio Mayor de Cundinamarca. En: Tabula Rasa Vol. 7. pp. 231-249.

Baker, C. (2011). Foundation of bilingual eduration and bilingualism ( $5^{\text {th }}$ Edition). Clevedon: Multilingual Matters.

Bialystok, E., Luk, G., Peets, K. F. \& Yang, S. (2010). Receptive vocabulary differences in monolingual and bilingual children. En: Bilingralism Languageand Cognition Vol. 13, No 4. pp. 525-531.

Calderón Ibáñez, A. \& Q uijano Peñuela, J. (2010). Características de comprensión lectora en estudiantes universitarios. En: Reista Estudios soiojuńdicos Vol10, No 1. pp. 34-55.

Cassany, D. (2006). Taller detextos Barcelona: Paidós 
Charaudeau, P. (1992). Grammiredu sens \& del'expression Paris: Hachette.

Chimbutane, F. (2011).Rethinkingbilingual educationin Postcdanial Contexts O ntario, Canada: Multilingual Matters.

Cummins, J (2005). La hipótesis de la interdependencia 25 años después: la investigación actual y sus implicaciones para la educación bilingüe. En D. Lasagabaster, D. Lasagabaster-Herrarte \& J.M Sierra (Eds.), Multiling̈̈ismoy miltialturalismo en la ๕ada (pp. 113-132). Barcelona: Horsori Editorial, S.l.

De Landsheere, G. (1973). Letęt dedosure Mesuredelalisibilitéđ dela comprehension Paris : Fernand Nathan.

Efklides, A. (2009). The role of metacognitive experiencies in the learning process. En: Psicothema, Vol. 21. pp. 76-82.

Galindo, A. (2009). Bilingüism, habilidades matalingǘsticas ylenguajecscito A cacamientotérico experimental. Armenia: Editorial Kinesis.

Genesee, F. (2004). What do we know about bilingual education for majority language students. En T. K. Bhatia \& W. Ritchie (Eds.), Handbook of bilingualism and multiculturalism (pp.547-576). Malden, MA: Blackwell.

Genesee, F., \& G eva, E. (2006). Cross-linguistic relationships in working memory, phonological processes, and oral language. En D. August \& T.

Shanahan (Eds.), Repart of theNational Literagy Pand onK-12 YauthandAddescents (pp. 169177). Mahwah, NJ: Lawrence Erlbaum Associates.

Genesee, F., \& Jared, D. (2008). Literacy development in early French immersion programs. En: Canadian Psydhdogist, Vd.49,No 2. pp. 140-147.

Grosjean, F. (2010). Bilingual: Lifeand reality. Cambridge: Mass Harvard.

Hamers, J.F. \& Blanc, M. (2000). Bilinguality and bilingualism Cambridge: Cambridge University Press.

Morais, J. (2001). El artedeler. Madrid: A. Machado Libros, S.A.

Mueller G athercole, V. C. (2013). Issues in the assessment of bilinguals Ontario, Canada: Multilingual Matters.

Muñoz, C. (2002). Aprenderidiamas Barcelona: Ediciones Paidós S. A.

Padilla, C., D ouglas, S., Lopez, E. (2011). Taller deprácticas de comprenión y producción de textos argumentativos Cordoba: Comunicarte.

Peal, E. y Lambert, W. E. (1962). The relation of bilingual to intelligence. E n: Psydhdogical Monogaphs Vol. 76, No27. pp. 1-23. 
Formación bilingüe en licenciatura de Lenguas Modernas y comprensión lectora

Perelman, C. \& Olbrechts-Tyteca (1958/ 1992). Traitédel'argumetation(5éd.). Bruxelles : Éditions de l'Université de Bruxelles.

Sierra, J. (1993). Revisando algunos aspectos de la educación bilingüe. En: Commicadón, Lenguajey Educacón Vol. 17. pp. 29-40.

Thyrion, F. (1997). L'éeit argumenté Questions d’apprentissage Louvain-la-Neuve : Peeters.

Trueta, Barrachina y Pascual (2012). Resultadbs dd moddolingǘsticodeCataluña. La eidenaia emṕnica Cataluña: Universitat O berta de Catalunya.

Turnbull, M., Lapkin, S., Hart, D. (2001). G rade 3 immersion students' performance in literacy and mathematics: Province-wide results from O ntario (1998-1999). En: Camadian Modem LanguageReview Vol. 58, No 1. pp. 234-258.

Van der Walt, C. (2013). Multilingual higher eduration Clevedon:Multilingual Matters.

Weir, C.J. (1990). Commuicativelanguagetesting London: Prentice Hall. 